\title{
A Hierarchical EMS for Aggregated BESSs in Energy and Performance-based Regulation Markets
}

\author{
T. Zhang, Student Member, IEEE, S. X. Chen, Member, IEEE, H. B. Gooi, Senior Member, IEEE \\ and J. M. Maciejowski, Fellow, IEEE
}

\begin{abstract}
The battery energy storage systems (BESSs) have been increasingly installed in the power system, especially with the growing penetration rate of the renewable energy sources. However, it is difficult for BESSs to be profitable due to high capital costs. In order to boost the economic value of BESSs, this paper proposes a hierarchical energy management system (HiEMS) to aggregate multiple BESSs, and to achieve multi-market business operations. The proposed HiEMS optimizes the multi-market bids considering a realistic BESS performance model, and coordinates the BESSs and manages their state of charge (SOC) values, according to their price penalties based on dynamically generated annualized cost. By taking part in the energy market and regulation market at the same time, the cost-performance index (CPI) of the BESS aggregation is greatly improved. The impact of photovoltaic generation (PV) on system performance and CPI is also studied.
\end{abstract}

Index Terms-Battery energy storage system (BESS), energy management system (EMS), electricity market, regulation market

\section{NOMENCLATURE}

$\begin{array}{ll}\text { A. Indices } & \\ i, i j & \text { BESS group index and individual BESS } \\ & \text { index in the } i^{t h} \text { group. } \\ t, t^{\prime} & \text { Time step index for the schedule optimizer } \\ & \text { and dispatch optimizer. } \\ s & \text { Scenario index. } \\ m & \text { Segment index for piecewise linearization. }\end{array}$

\section{B. Parameters}

$H_{p}, H_{p}^{\prime}$

$P_{i}^{\min }, P_{i j}^{\min }$

$E_{i}^{\max }, E_{i j}^{\max }$

$c_{e, t}^{s}$

$c_{b a t, i}, c_{b a t, i j}$

$c p_{t}^{s}, p p_{t}^{s}$

$p^{s}$
$P_{t}^{P V}, \bar{P}_{t}^{P V}$

$P_{t}^{\text {load }}, \bar{P}_{t}^{\text {load }}$

$$
\begin{gathered}
P_{t}^{r e g} \\
h_{e}, h_{r} \\
\text { C. Variables } \\
P_{i, t}^{e, d}, P_{i, t}^{e, c} \\
P_{i, t}^{r} \\
P_{i j, t}^{d}, P_{i j, t}^{c} \\
\bar{P}_{t}^{\text {grid }}, P_{t}^{\text {grid }}
\end{gathered}
$$

$E_{i, t}, E_{i j, t}$

$h_{t}, h_{m, t}$

$U_{m, t}$
Real-time regulation signal from the regulation market at time $t$.

Time interval in markets (hour) and time duration for regulation signals (seconds).

Dispatched group discharge and charge for the $i$ th BESS group at time $t$ (MW).

Power bid for group $i$ at time $t$ in regulation market (MW).

Real-time discharge and charge for each BESS (MW) at time $t$.

Scheduled hourly power consumption from the energy market and the real-time consumption (MW).

Energy stored at time $t$ for the $i$ th BESS group and the $i j$ th BESS (MWh).

Regulation energy scheduled at time $t$ and scheduled regulation energy at time $t$ when it falls to segment $m$ (MWh/MW).

Binary variable to represent whether the BESSs operate in the $m$ th segment.

\section{INTRODUCTION}

B ATTERY energy storage systems (BESSs) are gaining increasing research interests in power system applications, for their fast-response ability and control flexibility. Nonetheless, some recent BESS studies state that it is difficult for BESSs to be profitable due to high battery costs [1], even with the proposed optimal sizing scheme [2].

In order to promote the economic value of BESSs, BESSs are maneuvered to participate in market operations. Reference [3] provides a thorough optimal bidding framework for BESS's energy market participation. However, for BESS's multiple-market participation, there are mainly two issues described in the following two paragraphs.

Firstly, an optimal coordination between multiple-market operations is required. A number of papers have studied the optimization formulations based on multiple available markets. Reference [4] optimizes the BESS bidding in the day-ahead energy and spinning reserve markets, and reference [5] further includes the ramp-up and ramp-down frequency regulation service in the revenue. Moreover, a more financially rewarding performance-based regulation service is considered by [6]. In [6], the amount of energy consumed by regulation is set as a constant with a large margin (15 minutes or $0.25 \mathrm{MWh} / \mathrm{MW}$ ) 
to ensure the high performance (above 0.9 out of 1), while the actual energy requirement remains uncertain. In real market revenue accounting, the final regulation revenue is proportional to the performance as well as the power bids. Therefore, when scheduling the energy capacity of BESS, keeping a large energy margin will limit the amount of power that BESS can bid into the markets; in other words, there is a trade-off between the performance and the bids. A constant regulation energy schedule cannot fully utilize the BESS capacity and will fail to get the optimal reward.

Secondly, due to the minimum capacity requirement from the market regulator, several BESSs need to be aggregated to reach the minimum threshold to enter the market. When multiple BESSs are connected in the same network, coordination between the units helps to fully exploit the aggregation storage capacity, and optimize the real-time operation. In the current literature, the coordination of multiple BESSs is not sufficiently addressed. In [7], conventional "master-slave" logic is used to coordinate different BESSs in the aggregation. This method will result in premature aging of the "master" battery, and might introduce an extra replacement cost during the project. As such, the boost of capital return cannot be attained. A second method is proposed by [8], where the control signal distributed to each BESS is proportional to the ratio of its rated power to the overall power of multiple BESSs. This method does not consider the respective state of charge (SOC), hence resulting in non-response time from some of the BESSs.

To deal with the two issues stated above, this paper proposes a hierarchical energy management system (HiEMS) for a network with multi-use BESSs, taking part in both energy and regulation markets. For such systems involving control signals on different time schemes, a hierarchical structure is advantageous as claimed by previous research studies $[9,10,11]$. The operational objectives are separated into sub-controllers by their execution periods. Each sub-controller may have its own control algorithm, and exchanges the required information with each other.

In this paper, the optimal schedule optimizer includes an innovative realistic BESS performance model with regard to the regulation energy schedule, to characterize the trade-off between the performance and the power bids. The schedule optimizer also models the pricing uncertainties in both markets according to their respective statistic characteristics. The cost-effectiveness of BESSs is analyzed for optimizations with and without the performance model. The cost-performance index (CPI) values provide a reference for BESS entities to decide the optimal sizing and market participation to maximize the income of the multi-use BESSs. The impact of PV integration is also studied.

The proposed HiEMS attempts to coordinate BESSs of different battery types, various SOC, and power and energy capacity. The SOC values will be regulated around the expected average SOC to prevent individual saturation or depletion. Price penalties derived from the dynamic annualized cost (DAC) are utilized to dispatch the BESSs, thus increasing the average battery lifetime.

The rest of this paper is organized as follows: Section II

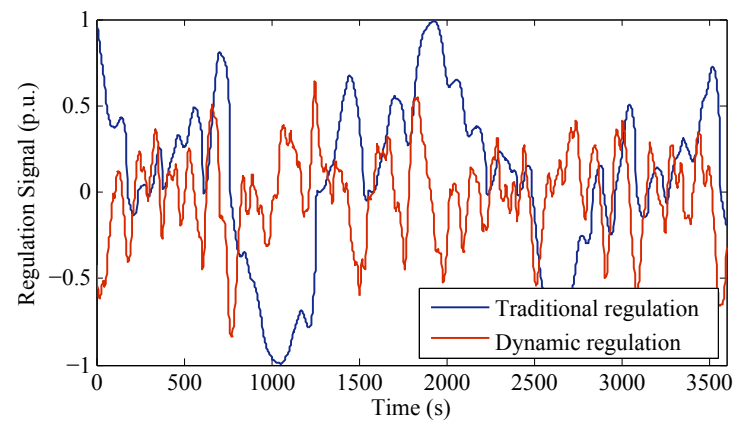

Fig. 1. Regulation signal samples for one hour.

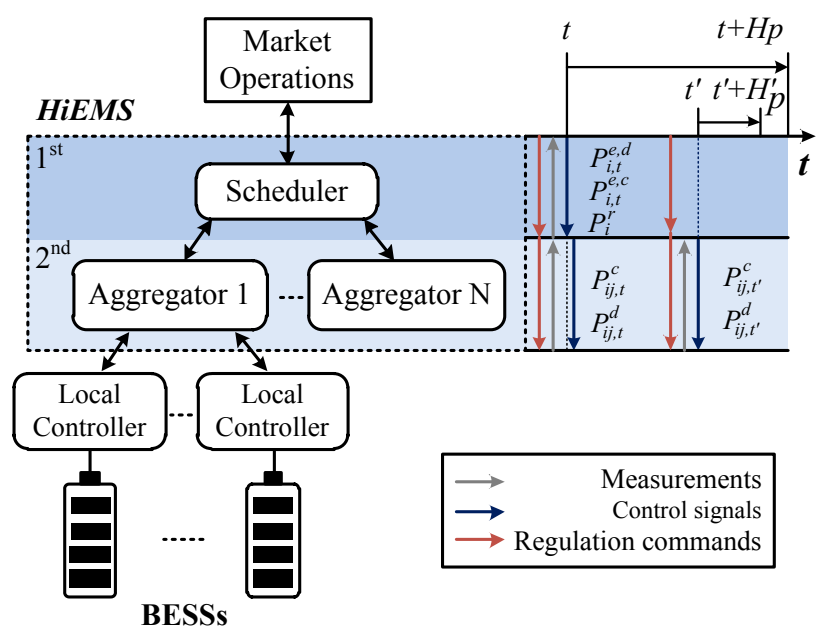

Fig. 2. Proposed HiEMS integration and its working time scheme.

introduces the HiEMS work flow and major functionalities. Section III presents the algorithm details in the optimizers. Section IV presents simulation studies, including optimality analysis and a complete analysis of results and their comparisons. Section V concludes the highlights of this paper.

\section{Proposed HiEMS CONFiguration}

\section{A. Market Mechanism and HiEMS Participation}

The National Energy Market of Singapore comprises a wholesale market and a retail market. To introduce competition in both markets, energy producers or consumers (prosumers) above a certain size threshold can sell or purchase electricity subject to the real-time pricing scheme. In the real-time market, the market clearing engine generates energy, reserve, and regulation prices every 30 minutes. Currently, regulation providers in the Singapore market are paid monthly based on the uniform regulation price, without performance standards.

However, with the increase penetration rate of intermittent renewable energy sources, the grid requires faster and larger amount of regulation capacity. In North America, PJM has introduced a performance-based regulation market, where participants can choose to respond to the slower-varying traditional regulation signal or the dynamic regulation signal. The signals shown in Fig. 1 are derived from the area control error (ACE) [12] in (1):

$$
A C E=\left(P_{\text {tie }}-P_{\text {tie }, \text { sched }}\right)+B_{f}(f-50)
$$




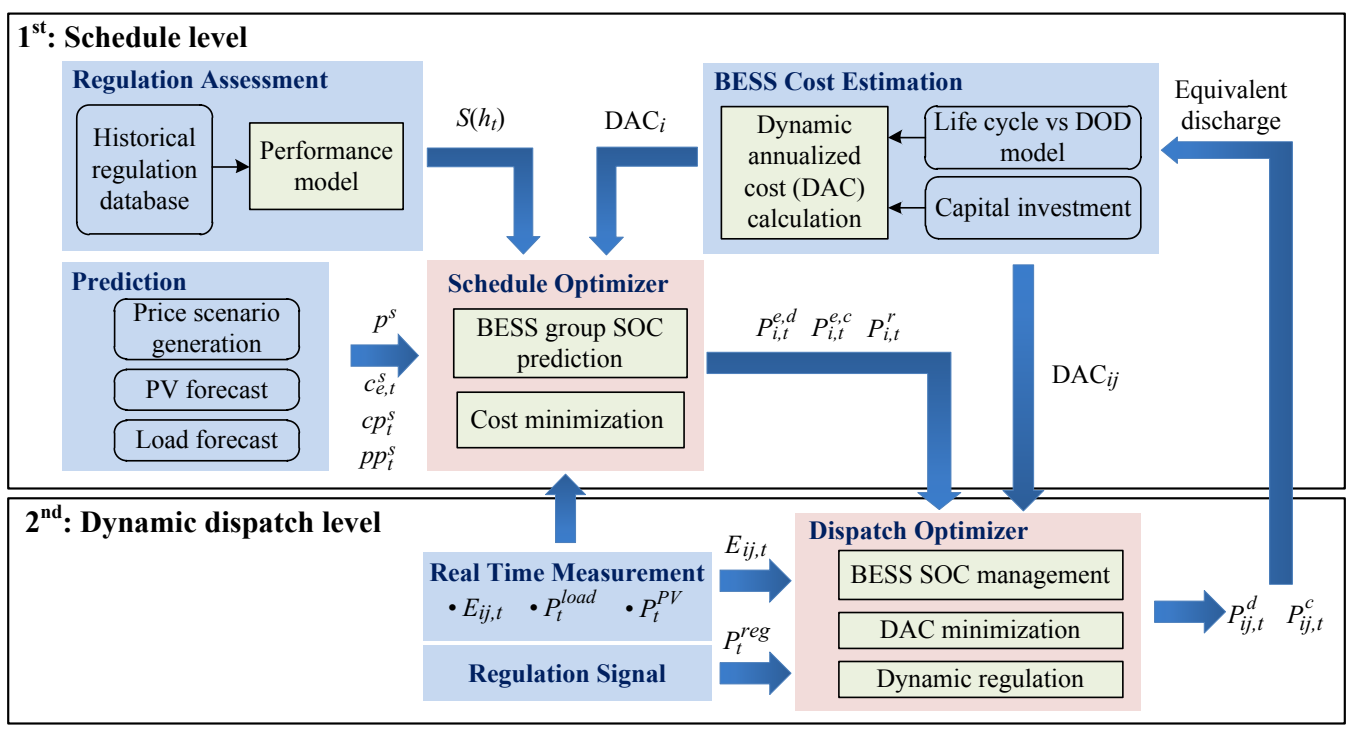

Fig. 3. Major function modules and data exchanges in the proposed HiEMS.

where $P_{t i e}$ is the real tie-line power; $P_{t i e, s c h e d}$ is the scheduled value provided by the system operator; $B_{f}$ is the frequency bias factor of the control area, which is an estimation of the system response characteristic in $\mathrm{MW} / \mathrm{Hz}$; and $f$ is the measured grid frequency.

The dynamic regulation signal is distributed to very fast responding units, and these units will be rewarded with higher payments [8]. Participating in the performance-based regulation is very profitable for BESS units, compared to participating in the energy market only [6, 13].

The HiEMS intends to incorporate energy market and performance-based regulation market participation. Although market clearing processes generate prices at the same time, the regulation market is sending real-time commands every few seconds. Therefore, the HiEMS works at two levels to comply with different control time schemes: 30 minutes or 1 hour for the market schedules and 2 seconds for the dynamic regulation.

The proposed HiEMS integration and its working time scheme are illustrated in Fig. 2. In the HiEMS, several BESSs in a nearby region are aggregated to participate in the markets as one participant. The coordination is conducted by the HiEMS, and thus no communication among the individual BESSs is required. The $1^{\text {st }}$ level generates regulation bids $P_{i}^{r}$ and energy set points $P_{i}^{e, c}$ and $P_{i}^{e, d}$ for the aggregators following the market time scheme; and the $2^{\text {nd }}$ level distributes individual set points $P_{i j}^{c}$ and $P_{i j}^{d}$ optimally according to real-time regulation commands and fluctuation in $\mathrm{PV}$ and loads.

This section introduces the work flow and the major functionalities of the proposed HiEMS illustrated in Fig. 3. The details of the optimizers are introduced in Section III.

\section{B. Schedule level}

The schedule level ( $1^{\text {st }}$ level) consists of four modules. The key module is the schedule optimizer, which is responsible for assessing the cost and revenue of the whole entity, and scheduling the network to achieve a greater economic value during the prediction horizon from time $t$ to $t+H_{p}$. The other three modules provide essential information for the decision making in the optimizer.

1) Prediction module: The Prediction module predicts PV and load levels, future market prices, and generates scenarios of possible realizations of the pricing information.

2) Regulation Assessment module: Regulation Assessment module is responsible for generating the BESS performance model. The frequency regulation units are not forced to perform strictly and rigidly, as long as a certain level of compliance is achieved. Therefore, simulation tests based on historical data are conducted to estimate the amount of energy needed to secure a certain level of performance over the long term. The simulation procedure is described in Fig. 4.

In this paper, the performance is evaluated using the PJM criteria [14]. The unit responses are measured on a 10-second basis, and the performance scores are averaged in every 5 -minute period. The final hourly average performance score $S$ consists of three sub-scores considering different aspects: precision sub-score $S_{P}$, delay sub-score $S_{D}$, and correlation sub-score $S_{C} . S_{P}$ measures the average errors between the regulation signal and the unit response. $S_{C}$ is the maximum value of the statistical correlations $\sigma_{t}$ between the 5-minute regulation signals and multiple sets of responses each with a 10 -second incremental shifting over 5 minutes. $S_{D}$ is calculated with the time $t_{\sigma}$ when the maximum correlation happens. $S$ is a weighted average of $S_{P}, S_{C}$ and $S_{D}$. The formulations provided by [14] are listed in (6):

$$
\begin{aligned}
& S_{P}=1-\frac{1}{N} \sum_{t=1}^{N}\left|\frac{y(t)-u(t)}{\bar{u}_{h}}\right| \\
& \sigma_{t}=\frac{\sum_{i=t}^{t+300 \mathrm{~s}}\left(u_{i}-\bar{u}_{h}\right)\left(y_{i}-\bar{y}_{h}\right)}{\sqrt{\sum_{i=t}^{t+300 \mathrm{~s}}\left(u_{i}-\bar{u}_{h}\right)^{2} \sum_{i=t}^{t+300 \mathrm{~s}}\left(y_{i}-\bar{y}_{h}\right)^{2}}} \\
& S_{C}=\max \left(\sigma_{t}\right) \quad t=[0,10 s, 20 s, \ldots, 300 s]
\end{aligned}
$$




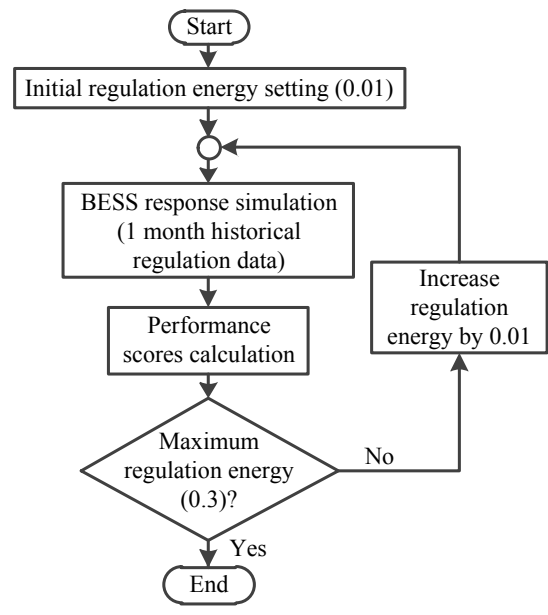

Fig. 4. Work flow for performance score simulations.

$$
\begin{aligned}
& S_{D}=\left|\frac{t_{\sigma}-300 s}{300 s}\right| \\
& S=A \cdot S_{P}+B \cdot S_{C}+C \cdot S_{D}
\end{aligned}
$$

where $y(t)$ is the actual unit response; $u(t)$ is the regulation signal; $\bar{u}_{h}$ is the rolling hourly average value of the regulation signal, and periods with $\bar{u}_{h}=0$ will not be included in the hourly average $S$ calculation; $N$ is the total number of sampled data, which is 360 in each hour; $\sigma_{t}$ is the correlation between the regulation signal and the unit response with $t$ seconds shifting; and parameters $A, B$, and $C$ are decided by the market governance. In most cases, their values are equal, i.e. $A=B=C=1 / 3$.

3) BESS Cost Estimation module: The schedule optimizer also considers the cost of BESS usage, which is derived from the dynamic annualized cost (DAC). The BESS DAC represents the annualized total BESS capital investment in relation to the on-line estimated battery lifetime $(L T)$. In this paper, the battery lifetime is estimated given the accumulated usage cycles and predefined depth-of-discharge (DOD).

The BESS Cost Estimation module stores the battery life cycle model, with life cycle data and varying DOD. After reading the DOD measurement, the expected life cycle can be determined from the model. At the same time, the usage cycles during time $t$ can be updated using the battery output $P^{c}$ and $P^{d}$ measured with a time step of $\Delta t$, based on the rated energy capacity $E_{B E S S}$ as in (7). The average battery lifetime (years) for each group $(L T)$ can be estimated in (8) using total usage cycles.

$$
\begin{aligned}
& \text { Usage cycle }=\sum_{t} \frac{\Delta t}{2 \cdot E_{B E S S}}\left(P^{c}+P^{d}\right) \\
& L T=\text { Life cycle/Usage cycle }
\end{aligned}
$$

The BESS capital cost data is also stored in this module. For BESSs with rated power $P_{B E S S}$ and rate energy $E_{B E S S}$, the cost consists of power investment $c_{M W}(\$ / \mathrm{MW})$ and energy investment $c_{M W h}(\$ / \mathrm{MWh})$ as in (9). The $D A C$ equals the total cost multiplying the capital recovery factor $C R F$ in (10) and (11), where the interest rate $r$ is set as 0.06 . The $D A C_{i}$ and $D A C_{i j}$ is proportionally downscaled to get the $c_{b a t, i}$ and $c_{b a t, i j}$.

$$
\begin{aligned}
& \text { Total cost }=c_{k W} \cdot P_{B E S S}+c_{k W h} \cdot E_{B E S S} \\
& C R F=\frac{r \cdot(1+r)^{L T}}{(1+r)^{L T}-1} \\
& D A C=\text { Total cost } \cdot C R F
\end{aligned}
$$

\section{Dynamic dispatch level}

The dynamic dispatch level $\left(2^{\text {nd }}\right.$ level) deals with set point distribution. After receiving $P_{i, t}^{e, d}, P_{i, t}^{e, c}$, and $P_{i, t}^{r}$ from the $1^{\text {st }}$ level, the dispatch optimizer in the $2^{\text {nd }}$ level aims to distribute $P_{i j}^{d}$ and $P_{i j}^{c}$ to each BESS in the groups.

The objective of the dispatch optimizer includes three parts: minimizing the BESS cost using the $D A C_{i j}$ from the $1^{\text {st }}$ level, managing the stored energy to avoid saturation or depletion of any BESSs, and responds to the real-time PV and load fluctuations, as well as operator's regulation command $P_{t}^{r e g}$ during the prediction horizon from time $t^{\prime}$ to $t^{\prime}+H_{p}^{\prime}$.

\section{OPTIMIZER FORMULATIONS}

In this section, the optimizers in the $1^{\text {st }}$ and $2^{\text {nd }}$ levels are described in details. As necessities for energy and regulation market participation, appropriate telecommunication and control devices will be installed, and provide access to up-to-date measurements. Therefore, the optimizers can be implemented using the predictive control scheme to provide better real-time performance.

\section{A. $1^{\text {st }}$ Level Schedule Optimizer}

As presented in Section II.B, the scheduling objective function $V_{1}$ aims to schedule the BESS aggregators to maximize the overall profit, which consists of the three parts: electricity bill from the energy market, revenue from the regulation market, and the BESS usage costs. The objective function is defined as follows:

$$
\begin{aligned}
V_{1}:=\min _{P_{i, t}^{e, d}, P_{i, t}^{e, c}, P_{i, t}^{r}} & \sum_{t}^{H_{p}}\left\{\sum_{s} p^{s}[\underbrace{c_{e, t}^{s} \bar{P}_{t}^{g r i d} h_{e}}_{\text {Energy market bill }}\right. \\
& -\underbrace{\text { Income } \left._{\text {reg,t }}^{s}\right]}_{\text {Regulation revenue }} \\
& +\sum_{i}^{n} \underbrace{\left[c_{b a t, i}\left(\frac{P_{i, t}^{e, c}+P_{i, t}^{e, d}}{2} h_{e}+P_{i, t}^{r} \bar{h}\right)\right]}_{\text {Battery cost }}\}
\end{aligned}
$$

where Income $\mathrm{reg}, \mathrm{t}_{\mathrm{t}, \mathrm{sepresents}}$ the regulation income at time $t$ in scenario $s, \frac{P_{i, t}^{e, c}+P_{i, t}^{e, d}}{2} h_{e}$ stands for the planned equivalent usage cycle for each group, and $\bar{h}$ represents the hourly average energy consumption $(0.13 \mathrm{MWh} / \mathrm{MW})$ by dynamic regulation service.

The regulation income has two components as shown in (13) and (14): $R C$ and $R P . R C$ is the capacity revenue for reserving the capacity, and $R P$ is the performance revenue.

$$
\text { Income }_{\text {reg, } \mathrm{t}}^{s}=R C_{t}^{s}+R P_{t}^{s}
$$




$$
\left\{\begin{array}{l}
R C_{t}^{s}=c p_{t}^{s} \cdot S\left(h_{t}\right) \cdot \sum_{i} P_{i, t}^{r} \\
R P_{t}^{s}=p p_{t}^{s} \cdot S\left(h_{t}\right) \cdot \mathrm{MR} \cdot \sum_{i} P_{i, t}^{r}
\end{array}\right.
$$

where $S\left(h_{t}\right)$ is the performance model, and MR represents the ratio between the requested mileage for the assigned resource (traditional or dynamic) to that of the traditional resources. MR further awards the dynamic resource for tracking longer mileage.

While BESS performance is usually assumed to be constantly high, the energy consumption by regulation service actually has an effect on the BESS score. This paper formulates a dynamic performance $S\left(h_{t}\right)$ with regard to the regulation energy $h_{t}$ for the regulation market.

The dynamic performance of BESS can be represented by a piecewise-linear function as in (15).

$$
S\left(h_{t}\right)=\sum_{m}\left(a_{m} \cdot h_{m, t}+b_{m} \cdot U_{m, t}\right)
$$

where $h_{t}=\sum_{m} h_{m, t}$, and $a_{m}, b_{m}$ are piecewise linearized coefficients for segment $m$.

The objective function is subject to the following constraints.

1) BESS Power rating and bid constraints:

$$
\begin{aligned}
& P_{i}^{\min }+P_{i, t}^{r} \leq P_{i, t}^{e, c}+P_{i, t}^{e, d} \leq P_{i}^{\max }-P_{i, t}^{r} \quad \forall i, t \\
& P_{i, t}^{r} \geq 0 \quad \forall i, t
\end{aligned}
$$

2) Power balance constraint:

$$
\bar{P}_{t}^{\text {grid }}=\bar{P}_{t}^{\text {load }}-\bar{P}_{t}^{P V}-\sum_{i}\left(P_{i, t}^{e, d}-P_{i, t}^{e, c}\right)
$$

3) Energy constraints: The average amount of stored energy of each group is predicted by coulomb-counting method [15] shown in (19), considering different charging and discharging efficiencies $\eta_{i}^{d}$ and $\eta_{i}^{c}$ for various battery groups.

$$
\begin{aligned}
E_{i, t}=E_{i, t-1} & -\underbrace{\frac{h_{e}}{\eta_{i}^{d}} \cdot P_{i, t}^{e, d}+\eta_{i}^{c} \cdot h_{e} \cdot P_{i, t}^{e, c}}_{\text {Energy arbitrage }} \\
& -\underbrace{\frac{\bar{h}}{\eta_{i}^{d}} \cdot P_{i, t}^{r}+\eta_{i}^{c} \cdot \bar{h} \cdot P_{i, t}^{r}}_{\text {Regulation energy consumption }}
\end{aligned}
$$

For each hour, the remained energy should be sufficient to maintain the schedule performance for providing both regulation up and down.

$$
E_{i}^{\min }+P_{i, t}^{r} \sum_{m} h_{m, t} \leq E_{i, t} \leq E_{i}^{\max }-P_{i, t}^{r} \sum_{m} h_{m, t} \quad \forall i, t
$$

To ensure continuous normal operation, the final energy stored is constrained to a certain value (50\% at the 24th hour).

$$
E_{i, 24}=0.5 E_{i}^{\max } \quad \forall i
$$

4) Discharge cycle constraints: The daily equivalent discharge cycle can be constrained to limit the usage of the battery as in (22) [3], where $\gamma$ is the number of allowed discharge cycles. A larger $\gamma$ allows more market participations, while a lower $\gamma$ restricts BESS bids. A proper $\gamma$ value can balance the economic profit and the BESS degradation.

$$
\sum_{t}^{24}\left[\frac{P_{i, t}^{e, c}+P_{i, t}^{e, d}}{2} h_{e}+P_{i, t}^{r} \sum_{m} h_{m, t}\right] \leq \gamma\left(E_{i}^{\max }-E_{i}^{\min }\right)
$$

5) Binary variable constraints:

$$
\sum_{m} U_{m, t}=1 \quad \forall t
$$

\section{B. 2nd level Dispatch Optimizer}

In the dispatch optimizer, the objective function includes three terms as shown in (24). The first term minimizes the battery DAC. The second term manages the stored energy levels by minimizing the squared absolute difference between the individual energy level and the scheduled group average level. The third term adjusts the real-time power output, by minimizing the deviation from the scheduled output caused by the load and PV fluctuations. The three objectives are combined by DAC, electricity price, and a weighting factors $\alpha_{1}$.

$$
\begin{aligned}
V_{2}:=\min _{P_{i j, t}^{c}, P_{i j, t}^{d}} \sum_{t}^{H_{p}^{\prime}} & \{\sum_{i} \underbrace{\sum_{j}\left[c_{b a t, i j}\left(P_{i j, t}^{c}+P_{i j, t}^{d}\right) h_{r}\right]}_{i^{t h} \text { group battery cost }} \\
& +\alpha_{1} \underbrace{\sum_{i} \sum_{j}\left|E_{i j, t}-E_{i}\right|^{2}}_{\text {Stored energy management }} \\
& +c_{e, t}^{s} \underbrace{\left(P_{t}^{\text {grid }}-\bar{P}_{t}^{\text {grid }}\right) h_{r}}_{\text {Real-time fluctuations }}\}
\end{aligned}
$$

subject to

1) Power balance constraint: The BESSs in each group are dispatched to satisfy the energy arbitrage goal as well as the required regulation power $P_{t}^{\text {reg }}$.

$P_{t}^{\text {grid }}-P_{t}^{\text {reg }}=P_{t}^{\text {load }}-P_{t}^{P V}-\sum_{i} \sum_{j}\left(P_{i j, t}^{d}-P_{i j, t}^{c}\right)$

2) Power rating constraints:

$$
P_{i j}^{\min } \leq P_{i j, t}^{c}+P_{i j, t}^{d} \leq P_{i j}^{\max } \quad \forall i, j
$$

3) Energy Constraints: The stored energy is updated in the same formula in (19) given a different time scheme.

$$
E_{i j}^{\min } \leq E_{i j, t} \leq E_{i j}^{\max } \quad \forall i
$$

\section{Simulation Studies}

\section{A. Simulation environment}

The proposed HiEMS is simulated based on a 14-bus Singaporean distribution system shown in Fig. 5. Bus 6 to bus 14 are load buses connected to residential buildings. The peak load of the distribution system is $1 \mathrm{MW}$. Each building 


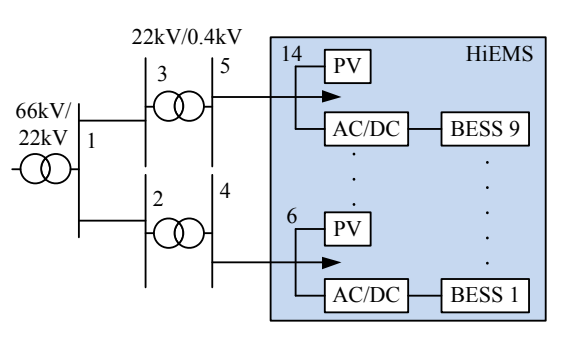

Fig. 5. A Singaporean 14-bus radial distribution network.

TABLE I

SETTINGS OF BESSS

\begin{tabular}{|c|c|c|c|c|c|c|c|c|c|c|}
\hline \multirow{2}{*}{\multicolumn{2}{|c|}{ BESS index }} & \multicolumn{4}{|c|}{ Vanadium group (V) } & \multicolumn{5}{|c|}{ Lithium group (L) } \\
\hline & & 1 & 4 & 5 & 6 & 2 & 3 & 7 & 8 & 9 \\
\hline \multicolumn{2}{|c|}{ Bus number } & 6 & 9 & 10 & 11 & 7 & 8 & 12 & 13 & 14 \\
\hline \multicolumn{2}{|c|}{ Power $(\mathrm{kW})$} & 120 & 80 & 100 & 80 & 40 & 20 & 40 & 20 & 20 \\
\hline \multicolumn{2}{|c|}{ Energy $(\mathrm{kWh})$} & 360 & 400 & 600 & 320 & 120 & 100 & 160 & 120 & 200 \\
\hline \multicolumn{2}{|c|}{$\mathrm{SOC}_{0}(\%)$} & 40 & 60 & 80 & 20 & 38 & 48 & 34 & 71 & 32 \\
\hline \multirow{2}{*}{ Efficiency } & $\eta_{d}$ & \multicolumn{4}{|c|}{0.88} & \multicolumn{5}{|c|}{0.9} \\
\hline & $\eta_{c}$ & \multicolumn{4}{|c|}{0.8} & \multicolumn{5}{|c|}{0.85} \\
\hline \multirow{2}{*}{\multicolumn{2}{|c|}{ Investment $\mid \frac{\$ / k W}{\$ / k W h}$}} & \multirow{2}{*}{\multicolumn{4}{|c|}{$\frac{600}{100}$}} & \multicolumn{5}{|c|}{1200} \\
\hline & & & & & & & & 600 & & \\
\hline
\end{tabular}

is equipped with a 75-kWp PV energy system accompanied by BESSs. Historical PV and load data is used in the case studies. The Uniform Singapore Energy Price (USEP) is used as the real-time buying and selling price, which applies to prosumers for all energy injections or withdrawals that occur in the Singapore power system. The market clearing engine generates the USEP half-hourly [16].

The BESSs are grouped by two widely used battery types: Vanadium (V group) and Lithium-ion (L group). Detailed BESS settings are listed in Table I. The discharge cycle constraint $\gamma$ is chosen as 2.5 for the $\mathrm{V}$ group and 1.4 for the $\mathrm{L}$ group. The dynamic performance is studied based on historical database. The piecewise-linear function parameters are obtained based on test results through curve fitting shown in Fig. 6.

The optimizers are modeled in General Algebraic Modeling System (GAMS), and the binary nonlinear optimizations can be solved by the KNITRO solver. The work flow and system simulation of HiEMS are built in Matlab. The optimizers and the simulation platform are interfaced using GDXMRW [17]. Prediction horizon $H_{p}$ and $H_{p}^{\prime}$ values are 24 hours and 2 seconds respectively.

\section{B. Scenario generation}

The uncertainty of energy and regulation prices is represented by a set of scenarios and their respective probabilities of realization. PV power and load consumption are considered deterministic in the analysis, and thus not included in the scenario generation.

1) Probability distribution: Regulation capacity and performance prices are highly unpredictable. Therefore, the scenarios are generated based on historical data. The distributions are plotted in Fig. 7(a) and Fig. 7(b). Average prices among all scenarios are plotted in Fig. 7(c).

For the USEP, future prices can be predicted by an artificial neural network trained using historical data. These point

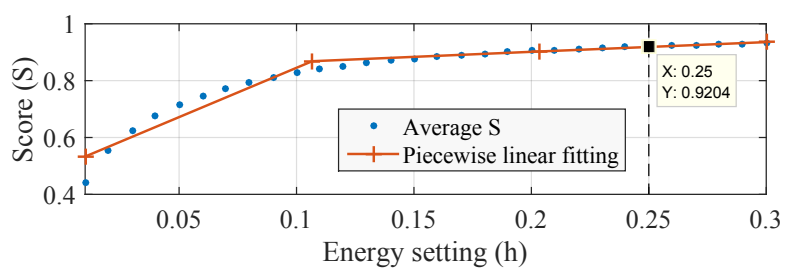

Fig. 6. Dynamic performance and piecewise linear fitting results.

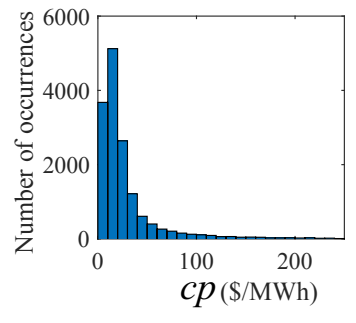

(a) Distribution of $c p$.

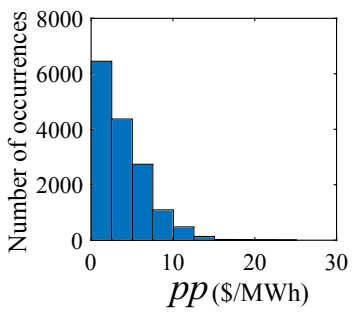

(b) Distribution of $p p$.

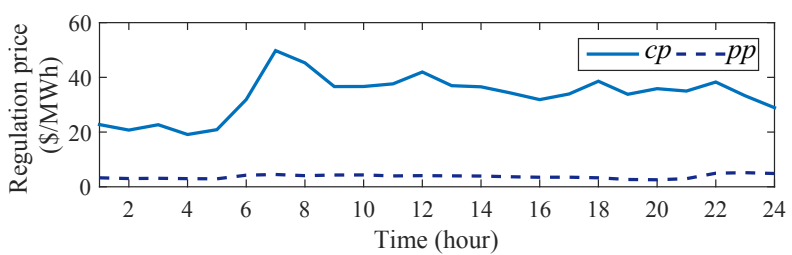

(c) Average regulation prices.

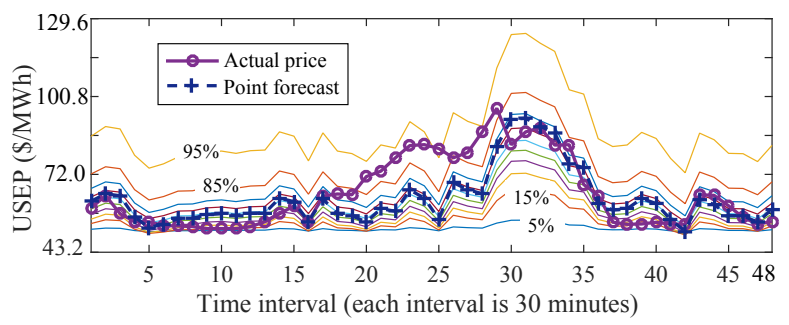

(d) Probabilistic forecast results for USEP.

Fig. 7. Regulation price distributions of a 638-day database.

forecast results can be expanded to probability forecast using quantile regression [18]. A sample of daily USEP probabilistic forecast is shown in Fig. 7(d). The USEP is averaged to a 24-hour time scheme in the optimizer.

2) Scenario generation and reduction: The Monte Carlo method is utilized to generate a large number of scenarios [19]. For each parameter at each time interval, a random number between $[0,1]$ is generated, and used to decided the parameter value according to the cumulative distribution generated above.

After generating the desired number of scenarios, the probability of each scenario is normalized so that the sum of all probabilities equals to 1 . To further lower the calculation burden, the numbers of scenarios can be reduced using a scenario reduction package SCENRED in GAMS.

\section{Optimality Analysis}

The optimization models proposed in (12) and (24) are multi-objective optimizations scalarized into single-objective ones. In the first level, the three objectives (electricity bill, regulation revenue, and battery cost), are commeasurable and are converted into a single-objective optimization problem 


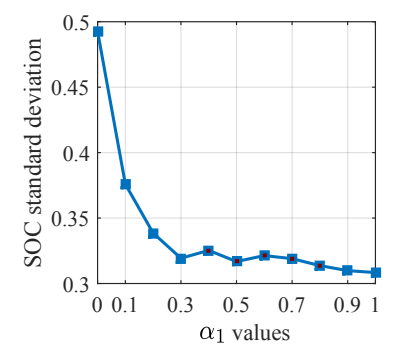

(a) Parametric studies for $\alpha_{1}$.

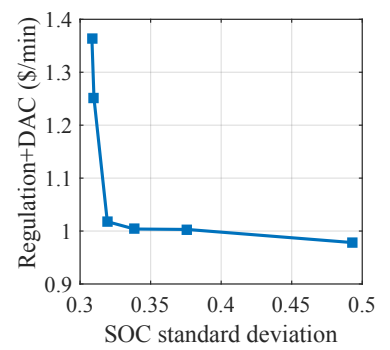

(b) Solution sets for $2^{\text {nd }}$ level optimizer.
Fig. 8. Regulation price distributions of a 638-day database.

weighted by their prices. To further enhance the economic gain from the markets, the battery cost has to be increased as a result of resulting more frequent BESS utilization; and to decrease battery cost, the BESS has to reduce market operations. In other words, The HiEMS cannot make more profit from the markets and decrease the DAC at the same time. Therefore, Pareto efficiency can be obtained in the optimization.

However, in the 2nd level optimizer, the SOC management objective is not measurable by economic values. The respective weighting usually complies with a preference decided by the HiEMS operator. In the parametric studies recorded in Fig. 8(a), the standard deviation of the SOC values can be reduced greatly with $\alpha_{1}$ larger than 0.2 . The $\alpha_{1}$ values are used in the optimality tests, and the equilibrium is shown in Fig. 8(b). A reduction of regulation penalty and DAC is traded off for an increase in SOC deviations from the expected value. In the simulations, $\alpha_{1}$ is chosen as 0.2 .

\section{Schedule optimizer studies}

1) Base case and comparisons: The $1^{\text {st }}$ level schedule optimizer results are shown in Fig. 9. The result in Fig. 9(a) is compared with the result of a constant optimizer using a constant regulation energy $h=0.25$ and score $S=0.92$, as shown in Fig. 9(b). The estimated state of charge curves are also recorded in Fig. 9(a) and Fig. 9(b). The dynamic regulation energy schedule $h_{t}$ and the respective expected scores $S$ are plotted in Fig. 9(c).

For both optimizers, the HiEMS bids most of its power into regulation market for most of the time. Charging activity is minimized only to maintain a sufficient SOC level. Lithium batteries are generally used less due to their high cost.

When compared to Fig. 9(b), the proposed dynamic optimizer in Fig. 9(a) manages to bid more power into regulation market, resulting from a more realistic and flexible regulation energy schedule. There are many hours when the dynamic optimizer is able to bid full power, while the constant optimizer can no longer provide power bidding because of stricter energy constraints such as hour 16 and 17.

In some intervals in Fig. 9(c), the regulation energy $h_{t}$ is less than the typical value of 0.25 . Although the resulting expected $S$ will be lower than the optimal value of 0.92 , the dynamic optimizer manages to boost the regulation income from more power bids, such as in hour 6,7 , and 8 . Conversely, in hour

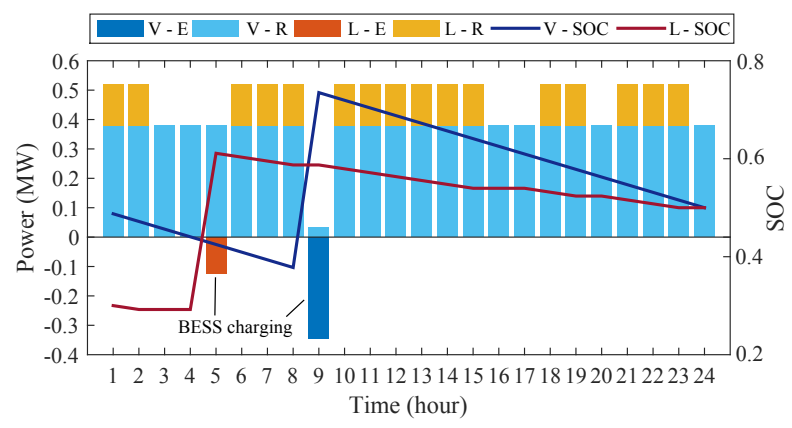

(a) Schedule Optimizer results (E stands for Energy market, R stands for regulation market).

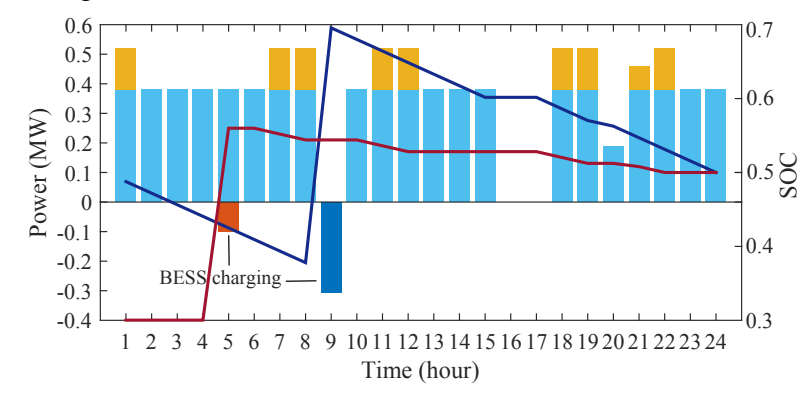

(b) Schedule Optimizer results with constant score.

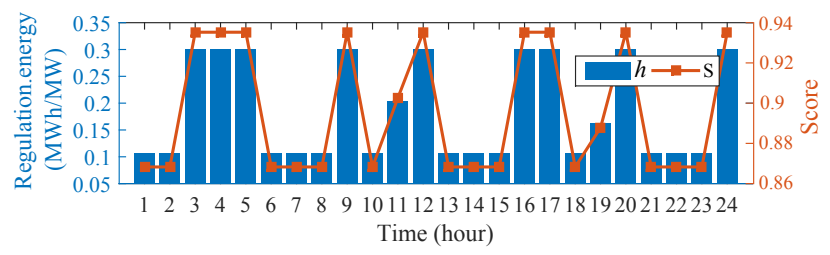

(c) Regulation energy schedule and expected performance scores.

Fig. 9. 24-hour schedules with dynamic performance and constant score.

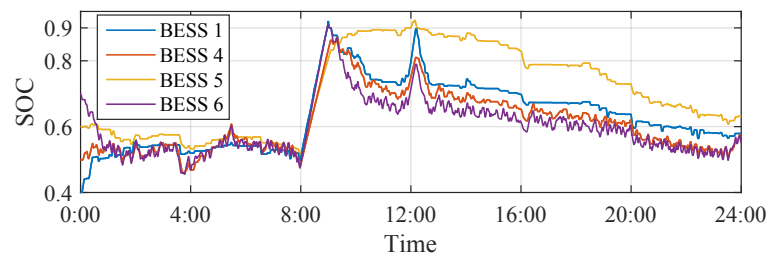

(a) $\mathrm{V}$ group SOC curves.

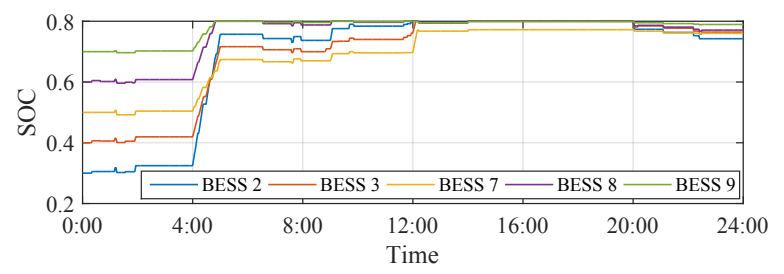

(b) L group SOC curves.

Fig. 10. 24-hour simulation SOC results.

3,4 , and 5 , the optimizer makes up for small power bids by reserving more $h_{t}$.

The SOC curves from a 24-hour test are plotted in Fig. 10. In the particular tested day, the actual regulation energy is even smaller than the dynamically scheduled regulation energy. As a result, the $\mathrm{V}$ group is already sufficient in responding the regulation signal, while the $\mathrm{L}$ group is often not utilized. 
TABLE II

COMPARISONS BETWEEN DYNAMIC AND CONSTANT SCORE

\begin{tabular}{|c|c|c|c|}
\hline & Dynamic & Constant \\
\hline \multicolumn{2}{|c|}{ Energy income (\$day) } & -34.56 & -30.02 \\
\hline \multirow{2}{*}{$\begin{array}{c}\text { Regulation } \\
\text { income (\$dday) }\end{array}$} & Capacity & 342.13 & 280.79 \\
\cline { 2 - 4 } & Performance & 114.11 & 93.81 \\
\hline \multicolumn{2}{|c|}{ Total daily income (\$/day) } & 421.68 & 344.57 \\
\hline \hline \multicolumn{2}{|c|}{ Total DAC (\$/year) } & 383,155 & 394,710 \\
\hline \hline \multicolumn{2}{|c|}{ CPI } & $40.17 \%$ & $31.88 \%$ \\
\hline
\end{tabular}

TABLE III

COMPARISONS BETWEEN DIFFERENT SCENARIO NUMBERS FOR A 238-DAY PERIOD

\begin{tabular}{|c|c|c|c|}
\hline \multicolumn{2}{|c|}{ Price information } & Actual income (\$) & Regret (\$) \\
\hline \multicolumn{2}{|c|}{ Perfect prediction } & 81,609 & N.A. \\
\hline \multirow{3}{*}{ Number of scenarios } & 10 & 75,888 & $-5,721$ \\
\cline { 2 - 4 } & 50 & 75,954 & $-5,655$ \\
\cline { 2 - 4 } & 100 & 78,383 & $-3,226$ \\
\hline
\end{tabular}

2) Income analysis: A detailed income breakdown is shown in Table II. While the dynamic optimizer pays more electricity bill for charging, it earns more revenue from the regulation market, and ends up with a higher overall daily income.

To further study the cost-effectiveness, cost-performance index (CPI) is calculated in Table II. The CPI represents the economic benefit returns from the investment as in (28).

$$
\mathrm{CPI}=\frac{\text { Earned Value }}{\text { Actual Cost }}=\frac{\text { Total annual income }}{\sum_{i} \mathrm{DAC}_{i}}
$$

The total earned value is estimated by multiplying the daily income by 365 days. The total actual cost for the BESSs is calculated based on the general pricing information from the literature $[20,21,22]$ is summarized in Table I.

By participating in the regulation market, BESS entities can greatly increase the CPI, while their CPI ranges from $4 \%$ to $15 \%$ when only providing energy arbitrage [4]. By dynamically adjusting the regulation energy schedule, the CPI can be further boosted to $40.17 \%$.

3) Impacts of scenario numbers: To study the long term effect of the scenario-based optimization, scheduling tests are carried out for a 238-day period with varying numbers of scenarios. For each day, required price scenarios are generated and used in the schedule optimizer. The real income of the calculated schedules are assessed with the actual price on a certain day. As a comparison, the perfect prediction case uses the actual prices in the optimizer, indicating that no forecast error is involved. The regret value is defined as the difference between the actual income and the income with perfect prediction.

According to the results summarized in Table III, optimization with more scenarios has a better representation of the possible price distribution, which is more robust in gaining a better income over a long period of time. However, more scenarios will greatly increase the computation burden. Therefore, a proper number of scenarios can be chosen considering the allowed computation time and the entity's risk tolerance.
TABLE IV

COMPARISONS BETWEEN THREE CONTROL METHODS

\begin{tabular}{|c|c|c|c|c|}
\hline \multicolumn{2}{|c|}{ Criteria } & \multicolumn{3}{c|}{ Controller schemes } \\
\cline { 3 - 5 } \multicolumn{2}{|c|}{} & HiEMS & PF & M-S \\
\hline \multicolumn{2}{|c|}{ Average performance score } & 0.92 & 0.90 & 0.82 \\
\hline \hline \multirow{2}{*}{$\begin{array}{c}\text { Average equivalent } \\
\text { usage cycles }\end{array}$} & V group & 1.98 & 1.42 & 1.46 \\
\cline { 2 - 5 } & L group & 0.23 & 1.20 & 1.22 \\
\hline \multicolumn{2}{|c|}{ Time of first replacement (years) } & 8.92 & 3.06 & 1.18 \\
\hline
\end{tabular}

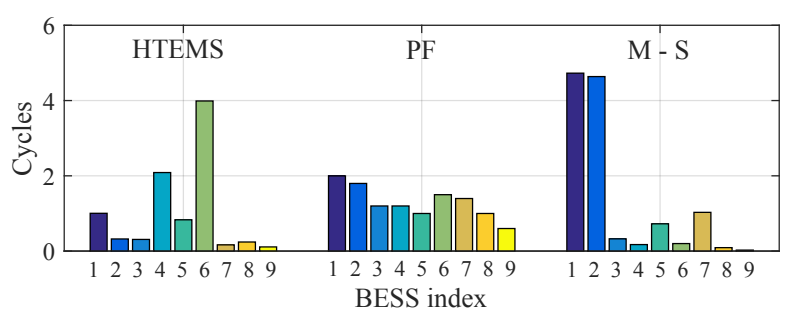

Fig. 11. Individual BESS equivalent usage cycles for three controllers.

\section{E. Dispatch optimizer performance and comparisons}

In this subsection, the HiEMS real time performance is compared with the participation factor method (PF) and the master-slave method (M-S). The test signal is the summation of the overall BESS set points taken from the base case study in Section IV.C.

The PF method [8] distributes the test signal to each BESS through its participation factor. Each participation factor is defined as the ratio of the individual BESS power rating to the total BESS power rating.

In the M-S method [7], the master BESS handles most of the commands, and the slave BESSs will only respond when the master reaches its SOC limit. The BESSs with the largest power ratings in group $\mathrm{V}$ and $\mathrm{L}$ are set as the masters in each group. The priority sequence in the $\mathrm{V}$ group is BESS $1>$ $5>4>6$, with BESS 1 as the master; and the sequence in the L group is BESS $2>7>3>8>9$, with BESS 2 as the master. In all the controllers, one BESS will cease to respond when its SOC exceeds the permitted range.

1) Real-time performance: As recorded in Table IV, the average performance score of HiEMS is $0.92,1.02$ times higher than 0.9 by PF, and 1.12 times higher than 0.82 by M-S.

2) BESS usage and lifetime: The equivalent usage cycles (UC) of the BESSs during the simulated time period is calculated by the formula in (8). The results are plotted in Fig. 11.

In general, HiEMS uses cheaper BESSs more frequently, and thus limiting the $\mathrm{L}$ group usage due to their high costs. The average $\mathrm{UC}$ of the $\mathrm{L}$ group is 0.192 times that of PF group and 0.189 times that of M-S group; while the average UC of the $\mathrm{V}$ group increases to 1.4 times that of $\mathrm{PF}$ group and 1.35 times that of M-S group.

In comparison to HiEMS, PF uses larger BESSs more often. For instance, the UC values of BESS 2 and 7, which have the largest power rating $(40 \mathrm{~kW})$ in the L group, are around 6 and 8 times larger than those of HiEMS. The UC of BESS 2 increases from 0.32 to 1.79 cycles, and that of BESS 7 increases from 0.17 to 1.39 cycles. 


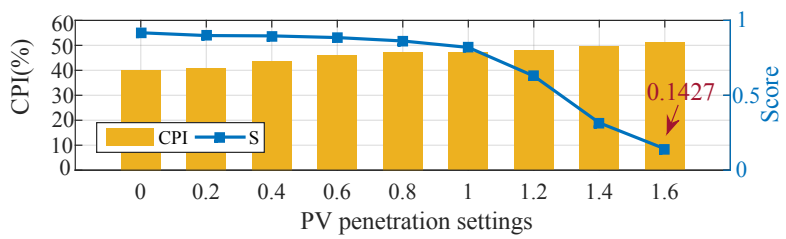

Fig. 12. CPI and score for different PV penetration settings.

For M-S, the master BESSs are over-burdened. The equivalent UC for BESS 1 is 4.73 cycles, while that of HiEMS and PF is 1.0 and 1.9 cycles respectively. Similarly, the equivalent UC for BESS 2 is 4.64 cycles, which is 14.5 times larger than 0.32 cycles by HiEMS and 2.59 times larger than 1.79 cycles by PF.

In practice, the minimum lifetime represents the first time when replacement is needed. The lifetime is estimated using the formulation in (8). The battery life cycle is chosen as 13,000 for the $\mathrm{V}$ group and 2,000 for the L group [22]. The capacity loss caused by self-discharge and internal leakage is ignored. As recorded in Table IV, the first replacement time with HiEMS is 2.92 times longer than that of PF, and 7.56 times longer than that of M-S.

\section{F. Impact of $P V$ penetration}

The impact of PV integration is simulated with different penetration settings. The original setting refers to $67.5 \%$ of the peak load power. The resulting CPI and score values are plotted in Fig. 12. With increased PV penetration, the CPI will be improved due to the PV power output. However, when the penetration rate grows to 1.6 times the original setting, the BESSs are not able to deal with the fluctuations. The system frequency regulation performance drops to 0.1427 . According to market rules, the entity will not be rewarded for that hour when the hourly score is lower than 0.25 [23]. Furthermore, the regulation entity will be disqualified if its 100-hour historical score drops below 40\% [14]. Therefore, the maximum CPI for this system can be boosted to no larger than $51.11 \%$ with PV generation.

\section{CONCLUSIONS}

This paper proposes a HiEMS for multi-use BESSs to improve their cost-effectiveness. Several BESSs can be aggregated and coordinated to participate in both the energy market and the regulation market. Multiple objectives are incorporated into two optimizers. Both can be implemented utilizing the predictive control strategy.

Realistic regulation performance is modeled to guarantee the optimal regulation energy schedule, and thus gaining maximum profit. After bringing in multi-market participation and considering the trade-off between the performance and power bids, the proposed HiEMS boosts the CPI to $40.17 \%$, compared to around $10 \%$ from only energy market participation, and $31.88 \%$ with constant score assumption. In terms of real-time performance, the dispatch optimizer coordinates the BESSs and manages the SOC. The HiEMS scores 0.92 , outperforms PF by 1.02 times and M-S by 1.12 times.
Cost awareness of the BESSs helps HiEMS manage BESS usage, and postpone the replacement investment. DAC is included as a part of the costs in both optimizers. This ensures that the control commands are sent to BESSs considering their specific battery characteristics. As a result, the time of first replacement for HiEMS is 2.92 times larger than that of the PF method and 7.56 times larger than that of the master-slave method.

Lastly, the CPI can be further improved by integration PV generation. However, the PV fluctuations will bring more challenge to the BESSs in maintaining an acceptable performance. In the tested system, the CPI maximum value can be no larger than $51.11 \%$ with increased PV generation.

\section{ACKNOWLEDGMENT}

This work is supported in part by DNV GL Energy (formerly KEMA) Technology Centre, Nanyang Technological University, and the Energy Innovation Research Programme (EIRP, Award No. NRF2014EWT-EIRP002-005), administrated by the Energy Market Authority (EMA). The EIRP is a competitive grant call initiative driven by the Energy Innovation Programme Office, and funded by the National Research Foundation (NRF) Singapore.

\section{REFERENCES}

[1] A. A. Hussein, N. Kutkut, Z. J. Shen, and I. Batarseh, "Distributed battery micro-storage systems design and operation in a deregulated electricity market," IEEE Trans. Sustain. Energy, vol. 3, no. 3, pp. 545-556, July 2012.

[2] Y. Yang, H. Li, and A. Aichhorn, "Sizing strategy of distributed battery storage system with high penetration of photovoltaic for voltage regulation and peak load shaving," IEEE Trans. Smart Grid, vol. 5, no. 2, pp. 982-991, 2014.

[3] H. Mohsenian-Rad, "Optimal bidding, scheduling, and deployment of battery systems in california day-ahead energy market," IEEE Transactions on Power Systems, vol. 31, no. 1, pp. 442-453, Jan 2016.

[4] J. M. Gantz, S. M. Amin, and A. M. Giacomoni, "Optimal capacity partitioning of multi-use customer-premise energy storage systems," IEEE Trans. Smart Grid, vol. 5, no. 3, pp. 1292-1299, May 2014.

[5] R. L. Fares, J. P. Meyers, and M. E. Webber, "A dynamic model-based estimate of the value of a vanadium redox flow battery for frequency regulation in texas," Applied Energy, vol. 113, pp. 189 - 198, 2014.

[6] G. He, Q. Chen, C. Kang, P. Pinson, and Q. Xia, "Optimal bidding strategy of battery storage in power markets considering performance-based regulation and battery cycle life," IEEE Trans. Smart Grid, vol. PP, no. 99, pp. 1-1, 2015.

[7] S. J. Lee, J. H. Kim, C. H. Kim, S. K. Kim, E. S. Kim, D. U. Kim, K. K. Mehmood, and S. U. Khan, "Coordinated control algorithm for distributed battery energy storage systems for mitigating voltage and frequency deviations," IEEE Trans. Smart Grid, vol. PP, no. 99, pp. 1-1, 2015.

[8] S. X. Chen, T. Zhang, H. B. Gooi, R. D. Masiello, and W. Katzenstein, "Penetration rate and effectiveness studies of aggregated bess for frequency regulation," IEEE Trans. Smart Grid, vol. PP, no. 99, pp. 1-1, 2015.

[9] A. Ulbig, M. Arnold, S. Chatzivasileiadis, and G. Andersson, "Framework for multiple timescale cascaded MPC application in power systems," in 18th IFAC World Congr., 2011, 2011, pp. 10472-10 480.

[10] A. Hooshmand, B. Asghari, and R. K. Sharma, "Experimental demonstration of a tiered power management system for economic operation of grid-tied microgrids," IEEE Trans. Sustain. Energy, vol. 5, no. 4, pp. 1319-1327, Oct. 2014.

[11] H. H. Abdeltawab and Y. A. I. Mohamed, "Market-oriented energy management of a hybrid wind-battery energy storage system via model predictive control with constraint optimizer," IEEE Trans. Ind. Electron., vol. 62, no. 11, pp. 6658-6670, Nov 2015.

[12] P. Kundur, Power system stability and control. New York: Mcgraw-Hill, 1993, ch. 11.1, pp. 606-610. 
[13] T. Zhang, H. B. Gooi, S. X. Chen, and T. Goh, "Cost-effectiveness studies of the besss participating in frequency regulation," in 2015 IEEE ISGT ASIA, Nov 2015, pp. 1-6.

[14] (2009) PJM Manual 12: Balancing Operations. [Online]. Available: www.pjm.com

[15] S. X. Chen, H. B. Gooi, and M. Q. Wang, "Sizing of energy storage for microgrids," IEEE Trans. Smart Grid, vol. 3, no. 1, pp. 142-151, March 2012.

[16] Energy market company: Guide to prices. [Online]. Available: https://www.emcsg.com/MarketData/GuideToPrices

[17] (2015) Matlab and GAMS: Interfacing Optimization and Visualization Software via the GDXMRW Utilities. [Online]. Available: http://www.gams.com/ steve/gdxmrw.html

[18] A. U. Haque, M. H. Nehrir, and P. Mandal, "A hybrid intelligent model for deterministic and quantile regression approach for probabilistic wind power forecasting," IEEE Transactions on Power Systems, vol. 29, no. 4 pp. 1663-1672, July 2014.

[19] T. Niknam, R. Azizipanah-Abarghooee, and M. R. Narimani, "An efficient scenario-based stochastic programming framework for multi-objective optimal micro-grid operation," Applied Energy, vol. 99, pp. 455 - 470, 2012. [Online]. Available: http://www.sciencedirect.com/science/article/pii/S0306261912003030

[20] C. Blanc, "Modeling of a vanadium redox flow battery electricity storage system," Ph.D. dissertation, Ecole Polytechnique Fédérale de Lausanne, 2009.

[21] H. Chen, T. N. Cong, W. Yang, C. Tan, Y. Li, and Y. Ding, "Progress in electrical energy storage system: A critical review," Progress in Natural Science, vol. 19, no. 3, pp. 291 - 312, 2009.

[22] "Front matter," in Advances in Batteries for Medium and Large-Scale Energy Storage, ser. Woodhead Publishing Series in Energy, C. M. S.-K. M. Lim, Ed. Woodhead Publishing, 2015, pp. i - iii.

[23] (2009) PJM Manual 28: Operating Agreement Accounting. [Online] Available: http://www.pjm.com//media/documents/manuals/m28.ashx

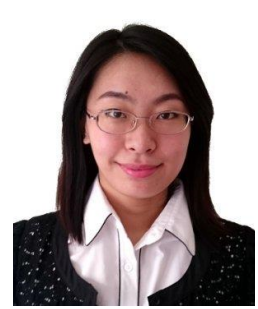

T. Zhang (GS'12) received her B.S. degree in Electrical Engineering and its Automation from Tianjin University, Tianjin, China. She is currently pursuing her Ph.D. degree at Nanyang Technological University, Singapore. Her research interests include smart energy management systems, virtual power plants, renewable energy sources and energy storage systems.

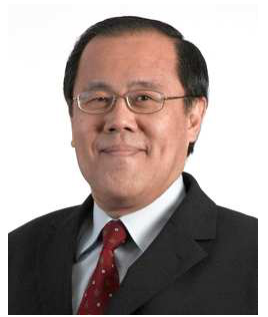

H. B. Gooi (SM'95) received his B.S. degree from National Taiwan University, M.S. degree from University of New Brunswick and Ph.D. degree from Ohio State University in 1978, 1980 and 1983 respectively. From 1983 to 1985, he was an Assistant Professor in the EE Department at Lafayette College, Easton, Pennsylvania, USA. From 1985 to 1991, he was a Senior Engineer with Empros (now Siemens), Minneapolis, Minnesota, USA where he was responsible for the design and testing coordination of domestic and international energy management system (EMS) projects. In 1991, he joined the School of Electrical and Electronic Engineering, Nanyang Technological University as a Senior Lecturer. Since 1999, he has been an Associate Professor. Since 2008, he has served as Deputy Head of Power Engineering Division. His current research focuses on microgrid energy management systems, electricity markets, spinning reserve, energy efficiency and renewable energy sources.

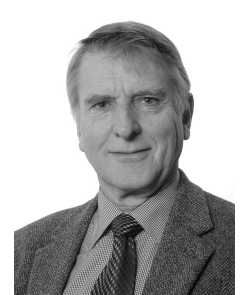

J. M. Maciejowski (M81SM96F11) received the B.Sc. degree in automatic control from Sussex University, Sussex, U.K., in 1971, and the Ph.D. degree in control engineering from Cambridge University, Cambridge, U.K., in 1978.

He was a Systems Engineer with Marconi Space and Defence Systems Ltd., Frimley, Surrey, U.K., from 1971 to 1974, working mostly on attitude control of spacecraft and high altitude balloon platforms. Since 1981, he has been with the University of Cambridge, Cambridge, where he is currently a Professor of Control Engineering and the Head in the Information Engineering Division. He was a President of the European Union Control Association from 2003 to 2005 and a President of the Institute of Measurement and Control in 2002. His current research interests include the theory and applications of predictive control, and its application to fault-tolerant control, in system identification, and in the control of autonomous systems.

Prof. Maciejowski was a recipient of the Honeywell International Medal from the InstMC in 2008.

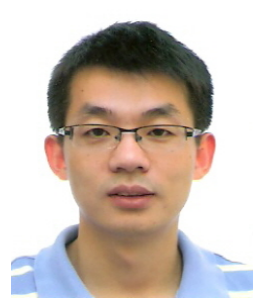

S. X. Chen (M'13) received his B.S. dual degree in Power Engineering and Business Administration from Wuhan University, China, M.S. and Ph.D degrees in Power Engineering from Nanyang Technological University, Singapore in 2007, 2008 and 2012 respectively. From 2012 to 2013, he was a research fellow of Energy Research Institute @ NTU, Singapore. Currently he works at DNV GL Energy (formerly KEMA). His research interests are smart energy management systems, energy efficiency, power system operation and planning, renewable energy sources and energy storage systems. 